\title{
Subcellular location prediction of proteins using support vector machines with alignment of block sequences utilizing amino acid composition
}

\author{
Takeyuki Tamura* and Tatsuya Akutsu
}

\author{
Address: Bioinformatics Center, Institute for Chemical Research, Kyoto University, Gokasho, Uji, Kyoto 611-0011, Japan \\ Email: Takeyuki Tamura* - tamura@kuicr.kyoto-u.ac.jp; Tatsuya Akutsu - takutsu@kuicr.kyoto-u.ac.jp \\ * Corresponding author
}

Published: 30 November 2007

BMC Bioinformatics 2007, 8:466
Received: 17 March 2007

Accepted: 30 November 2007

This article is available from: http://www.biomedcentral.com//47/-2/05/8/466

(c) 2007 Tamura and Akutsu; licensee BioMed Central Ltd.

This is an Open Access article distributed under the terms of the Creative Commons Attribution License (http://creativecommons.org/licenses/by/2.0), which permits unrestricted use, distribution, and reproduction in any medium, provided the original work is properly cited.

\begin{abstract}
Background: Subcellular location prediction of proteins is an important and well-studied problem in bioinformatics. This is a problem of predicting which part in a cell a given protein is transported to, where an amino acid sequence of the protein is given as an input. This problem is becoming more important since information on subcellular location is helpful for annotation of proteins and genes and the number of complete genomes is rapidly increasing. Since existing predictors are based on various heuristics, it is important to develop a simple method with high prediction accuracies.

Results: In this paper, we propose a novel and general predicting method by combining techniques for sequence alignment and feature vectors based on amino acid composition. We implemented this method with support vector machines on plant data sets extracted from the TargetP database. Through fivefold cross validation tests, the obtained overall accuracies and average MCC were 0.9096 and 0.8655 respectively. We also applied our method to other datasets including that of WOLF PSORT.

Conclusion: Although there is a predictor which uses the information of gene ontology and yields higher accuracy than ours, our accuracies are higher than existing predictors which use only sequence information. Since such information as gene ontology can be obtained only for known proteins, our predictor is considered to be useful for subcellular location prediction of newlydiscovered proteins. Furthermore, the idea of combination of alignment and amino acid frequency is novel and general so that it may be applied to other problems in bioinformatics. Our method for plant is also implemented as a web-system and available on http://sunflower.kuicr.kyoto-u.ac.jp/ -tamura/slpfa.html.
\end{abstract}

\section{Background}

Predicting subcellular location of proteins is one of the major problems in bioinformatics. This is a problem of predicting which part (e.g., Mitochondria, Chloroplast, etc.) in a cell a given protein is transported to, where an amino acid sequence (i.e., string data) of the protein is given as an input as shown in Fig. 1. This problem is becoming more important since information on subcellular location is helpful for annotation of proteins and genes and the number of complete genomes is rapidly 


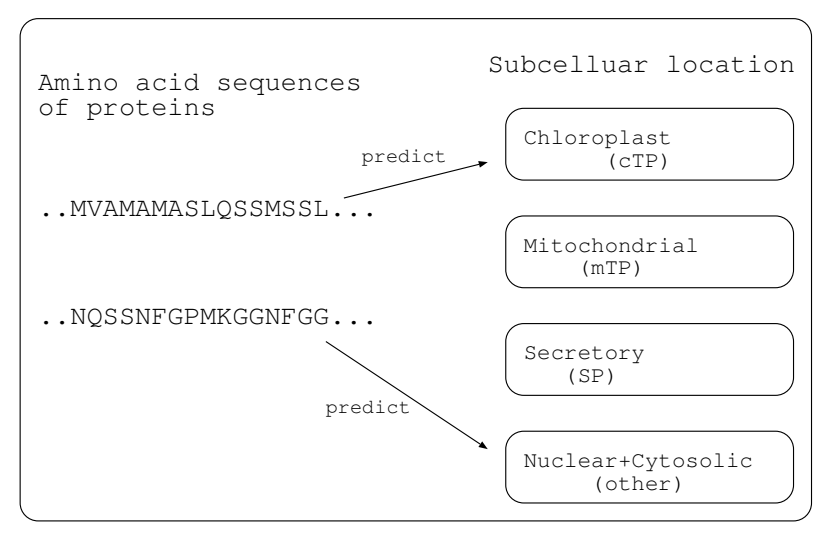

Figure I

Subcellular location prediction of proteins. Subcellular location prediction of proteins is a problem of predicting which part in a cell a given protein is transported to, where an amino acid sequence of the protein is given as an input.

increasing. Many methods have been proposed using various computational techniques. Furthermore, many webbased prediction systems have been developed based on these proposed methods.

PSORT $[1,2]$ is historically the first subcellular location predictor. PSORT and its major extension, such as WoLF PSORT $[3,4]$, use various sequence-derived features such as the presence of sequence motifs and amino acid compositions. Although there are many predicting methods, they can be roughly classified into two groups. One is the $\mathrm{N}$-terminal based method and the other is based on amino acid composition. TargetP [5] requires the N-terminal sequence as an input into two layers of artificial neural networks (ANN), utilizing the earlier binary predictors, SignalP [6] and ChloroP [7]. Reczko and Hatzigeorgiou used a bidirectional recurrent neural network with the first 90 residues in the $\mathrm{N}$-terminal sequence [8].

Cedano et al. developed ProtLock [9], which is based on the amino acid composition and the least Mahalanobis distance algorithm. Chou and Elrod used the covariant discriminant algorithm besides amino acid composition [10]. NNPSL [11] is an ANN-based method using the amino acid composition by Reinhardt and Hubbard. After the successful report by Reinhardt and Hubbard [11], application of machine learning techniques became popular in this field. A support vector machine (SVM) was implemented for SubLoc [12] instead of the ANN. Incorporating amino acid order as well as amino acid composition is expected to make it possible to improve prediction performance. The pseudo-amino acid composition was proposed by Chou [13] in order to deal with the effect of the amino acid order. Moreover, Cai and
Chou [14] have recently developed an accurate method integrating the pseudo-amino acid composition, the gene ontology information [15], and the functional domain composition [16]. Park and Kanehisa [17] developed an efficient method that incorporates compositions of dipeptides and gapped amino acid pairs besides the conventional amino acid composition. Yu et al.[18] successfully predicted localizations for Gram-negative bacteria by multiple feature vectors [19] based on $n$-peptide compositions. The concepts of the pseudo-amino acid and gapped amino acid pair compositions were merged in the residuecouple model proposed by [20]. Nair and Rost introduced LOCtree and showed mimicking the mechanism of cellular sorting yields good accuracies [21].

Recently, Matsuda et al.[22] proposed a novel representation of protein sequences. That representation involves local amino acid compositions and twin amino acids, and local frequencies of distance between successive (basic, hydrophobic, and other) amino acids. Each sequence is split into three parts: N-terminal, middle, and C-terminal in order to calculate the local features. The $\mathrm{N}$-terminal part is further divided into four regions for considering ambiguity in the length and position of signal sequences. It was combined with SVM for prediction of subcellular location of proteins. The results of computational experiments suggest that their method is one of the state-of-the-art methods. Though the prediction accuracy is high, the method is based on various heuristics. Furthermore, many of the heuristics are specific to the protein subcellular location problem. In this paper, we try to develop a less heuristic method for the protein subcellular location problem and improve the prediction accuracies. Our method does not use any knowledge of motifs, gene ontologies and other databases. Furthermore, feature vector is very simple, that is amino acid composition of 20 elements. Development of such a method is important since it may be applied to other problems in bioinformatics. For example, the spectrum kernel [23], which is a simple and general kernel function for SVM, has been applied to various problems including remote homology detection [23], recognition of DNA-binding proteins [24], prediction of protein-protein interactions [25] and prediction of protein subcellular location.

To develop a general and more accurate method, we combine two-techniques in a simple but novel and general way: sequence alignment and a feature vector based on amino acid composition. Although there are predictors (e.g., PSLpred [26], ESLpred [27]) which combine sequence similarity and SVM based method, alignment is applied directly to amino acid sequences. On the other hand, in our method as explained later, alignment is applied to block sequences, in which each block has an amino acid composition-based feature vector. Further- 
more, although there are some predictors which utilize sequence similarity and amino acid compositions (e.g. [28]), their method is a kind of hybrid procedure. That is, if sequence alignment can identify some homologous sequence, the prediction by sequence alignment is used. Otherwise, the prediction by SVM based method is used. Therefore, in their method, sequence alignment and SVM based method are independently executed. However, in our method, results of alignment are used as elements of kernel matrix.

It should be noted that amino acid composition-based feature vector is almost the same as spectrum kernel. Elements of our proposed kernel matrix are scores of alignment between sequences of substrings of proteins. The alignment scores are calculated in accordance with amino acid composition-based feature vectors as shown in Fig. 2

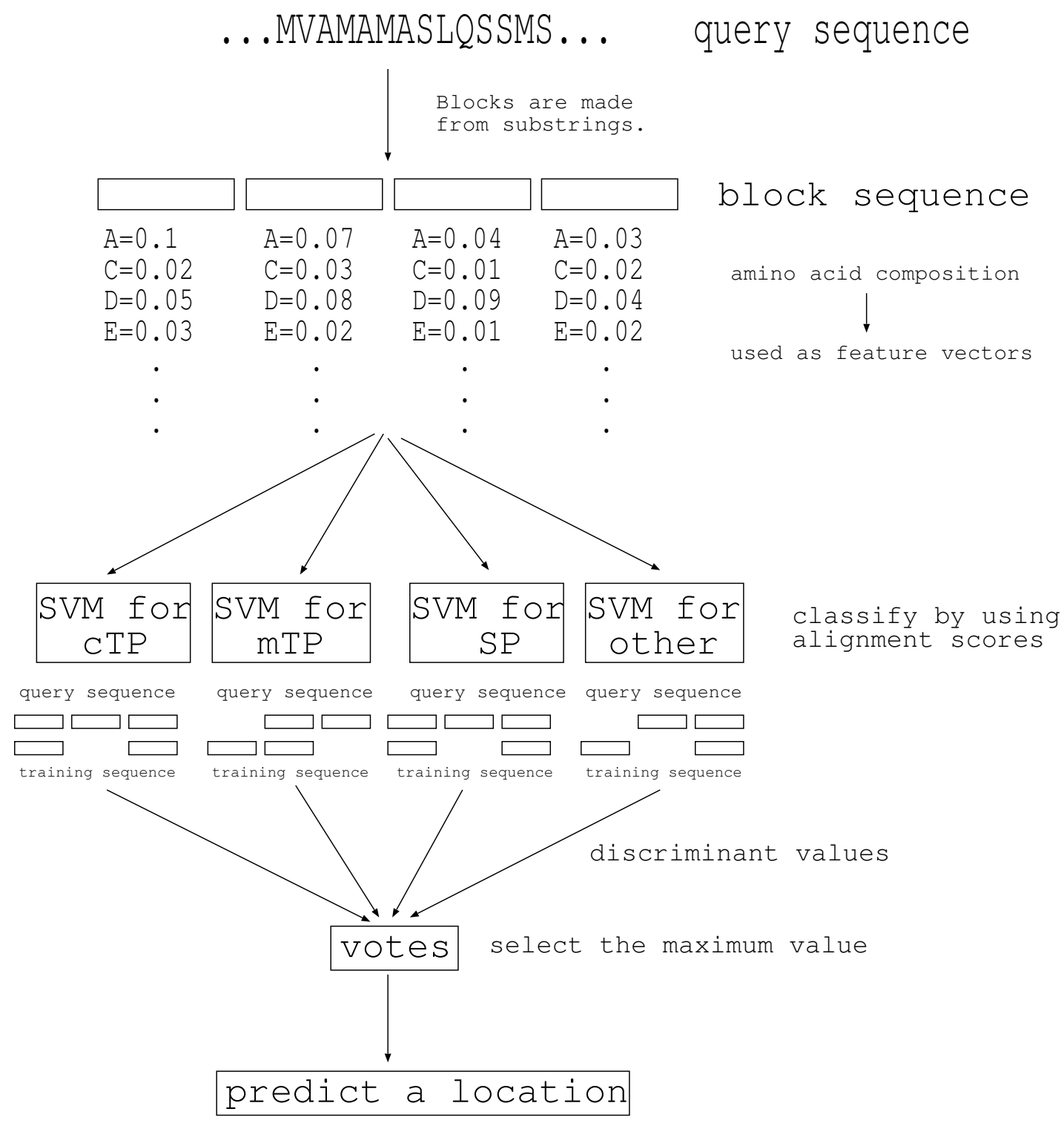

\section{Figure 2}

Overview of our method. Elements of our proposed kernel matrix are scores of alignment between block sequences of proteins. The alignment scores are calculated in which blocks are treated as if these were residues and the score between blocks is calculated from the corresponding feature vectors based on the amino acid frequency. 
(Details are explained later). As a result, we succeeded in improving prediction accuracies of the protein subcellular location problem for plant. To evaluate the prediction accuracies, we extracted plant data sets from TargetP data base and compared our method with existing methods through fivefold cross validation tests. Although our prediction method is less specialized for localization prediction than existing predictors, the overall accuracy and average MCC, which are standard measures of prediction accuracy, are 0.9096 and 0.8655 respectively. They are higher than existing predictors which use only sequence information. Furthermore, our method for plant is implemented as a web-system and available on [29].

Since the datasets obtained from TargetP is not very new, we also applied our method to plant datasets included in WoLF PSORT package version 0.2 that can be downloaded from [30]. As a result the overall accuracy of 0.8703 was obtained and this value is higher than that of WoLF PSORT.

\section{Results}

Table 1 shows the comparison of predictive accuracies on the TargetP plant data sets with existing methods which use only sequence information. It is seen that MCC of our proposed method for "cTP" is higher than those of the other three methods by $0.081 \sim 0.131$. It is also seen that MCC for "mTP" is higher than those of the other three methods by $0.051 \sim 0.104$. Since 0.05 is considered to be a large improvement, our approach seems to be suitable for predicting locations of plant proteins which destine for "cTP" and "mTP". On the other hand, improvements of MCC for "SP" and "other" are $0.028 \sim 0.041$ and 0.003 $\sim 0.080$ respectively. Although these improvements are not large, our sensitivity and specificity for "SP" are very high ( 0.9665 and 0.9319 respectively). Thus, our method is also suitable for predicting the location of proteins which destine for "SP" although the improvements are small. Therefore, our method succeeded in improving accuracies for "cTP" and "mTP" substantially and exceeding good accuracies of existing methods for "SP". However, our method is less effective at improving accuracies for "other" although our MCC for "other" is still higher than existing predictors in Table 1.

It is to be noted that our overall accuracy and average MCC are higher than any other predictor in Table 1. Furthermore, our MCC for each location is higher than that of any other predictor. Thus, it can be said that the prediction accuracy of our method is higher than existing predictors which use only sequence information.

Table 2 shows the values of parameters used in this experiment. Gap penalty denotes the penalty per gap in alignment between block sequences. That is, the score of -0.4 is given to a pair of a block and a gap. $\gamma$ is the parameter of RBF kernel. "posconstraint" is a parameter of GIST (see "Methods") which sets an explicit upper bound on the magnitude of the weights for positive training examples. Similarly, "negconstraint" sets an explicit upper bound on the magnitude of the weights for negative training exam-

Table I: Comparison of predictive accuracy for plant proteins in the TargetP data set. "cTP", "mTP", "SP", and "other" indicate proteins destined for chloroplast, mitochondria, secretory pathway, and other locations (nucleus and cytosol), respectively.

\begin{tabular}{|c|c|c|c|c|c|c|}
\hline Predictor & Location & Sensitivity & Specificity & MCC & Average MCC & Overall Accuracy \\
\hline \multirow[t]{4}{*}{ Our method } & cTP & 0.8440 & 0.9015 & 0.8507 & 0.8655 & 0.9096 \\
\hline & $\mathrm{mTP}$ & 0.9348 & 0.9125 & 0.8735 & & \\
\hline & $\mathrm{SP}$ & 0.9665 & 0.9319 & 0.9282 & & \\
\hline & other & 0.8148 & 0.8684 & 0.8095 & & \\
\hline \multirow[t]{4}{*}{ Matsuda et al. (2005) } & cTP & 0.7591 & 0.8474 & 0.7694 & 0.8244 & 0.8809 \\
\hline & mTP & 0.9240 & 0.8652 & 0.8227 & & \\
\hline & $\mathrm{SP}$ & 0.9219 & 0.9326 & 0.8983 & & \\
\hline & other & 0.8210 & 0.8586 & 0.8070 & & \\
\hline \multirow[t]{4}{*}{ Kim et al. (2004) } & cTP & 0.6874 & 0.8435 & 0.7222 & 0.7791 & 0.8479 \\
\hline & mTP & 0.8970 & 0.8392 & 0.7773 & & \\
\hline & SP & 0.8592 & 0.9428 & 0.8872 & & \\
\hline & other & 0.8027 & 0.7549 & 0.7296 & & \\
\hline \multirow[t]{4}{*}{ Emanuelsson et al. (2000) } & cTP & 0.85 & 0.69 & 0.72 & 0.79 & 0.853 \\
\hline & mTP & 0.82 & 0.90 & 0.77 & & \\
\hline & SP & 0.91 & 0.95 & 0.90 & & \\
\hline & other & 0.85 & 0.78 & 0.77 & & \\
\hline
\end{tabular}

Note: Parameters of Table 2 are used in our method. 
Table 2: Parameters used in the computational experiment. "Posconstraint" and "negconstraint" are parameters used in GIST.

\begin{tabular}{lcccccc}
\hline Location & Gap penalty & $\gamma$ of RBF & Posconstraint & Negconstraint & c & w \\
\hline cTP & 0.4 & 2.5 & 1.000000 & 0.009853 & 10 & 20 \\
mTP & 0.4 & 2.5 & 0.151072 & 0.099261 & 10 & 10 \\
SP & 0.4 & 2.5 & 0.032387 & 0.008566 & 10 & 20 \\
Other & 0.4 & 2.5 & 0.039955 & 0.008566 & 20 \\
\hline
\end{tabular}

ples. "c" and "w" are used to transform given protein sequences into block sequences as explained later.

Since data sets of TargetP are not very new, we also applied our method to plant data sets of WoLF PSORT, which are included in WoLF PSORT package version 0.2 and can be downloaded from [30]. We removed 35 proteins which have dual locations from original 2426 proteins. As a result, obtained average MCC and overall accuracy were 0.8343 and 0.8703 respectively. Although we have not implemented for data sets which include dual location proteins, our overall accuracy would still be 0.8557 even if our method predicted a false location for every dual location protein. Since the overall accuracy of WoLF PSORT is 0.86 , it seems that our method is also efficient for relatively new data sets. Details are shown in Table 3. Furthermore, we also applied our method to TargetP nonplant data sets. Details are shown in Table 4. Obtained average MCC and overall accuracy are 0.8452 and 0.9204 respectively. Although these values are slightly worse than those of Matsuda et al. [22], these are better than the other predictors of Table 4. Our MCC for MTP and "other" are worse than [22] by 0.022 and 0.005 respectively although our MCC for SP is slightly better than [22].

\section{Control experiments}

We executed some control experiments in order to evaluate the results of our method. Table 5 shows the comparison of our methods which use following feature vectors: (i) amino acid composition (ii) all adjacent amino acid composition (iii) amino acid composition and twin amino acid composition. "twin amino acid composition" is defined as the frequency of length two substrings "XX" for any amino acid X. For example, if an amino acid sequence "AAACC" is given, $\mathrm{AA}=0.5, \mathrm{CC}=0.25$ and the others $=0$ are obtained. As a result, (i) yielded the best result, $90.96 \%$ of overall accuracy. Overall accuracy of (ii) and (iii) were $73.72 \%$ and $90.64 \%$ respectively. Although twin amino acids were effective for the method of Matsuda et al.[22], they did not improve the overall accuracy of our method.

We also implemented the nearest neighbor classifier (NNC) with the similarity measure proposed in this manuscript. The obtained average MCC and overall accuracy were 0.7255 and 0.8128 . These values are worse than the SVM based method by 0.14 and 0.0968 respectively. Therefore, in the control experiment where the same similarity measure (our proposed method) and different classifiers (SVM and NNC) are used, SVM was better than

Table 3: Prediction accuracy for WoLf PSORT plant data sets. "Chlo," "cyto," "cysk," "E.R.," "extr," "golg," "mito," "nucl," "pero," "plas," "vacu" indicate, chloroplast, cytosol, cytoskeleton, endoplasmic reticulum, extracellular, Golgi apparatus, mitochondria, nuclear, peroxisome, plasma membrane, vacuolar membrane respectively.

\begin{tabular}{|c|c|c|c|c|c|c|c|}
\hline Predictor & Location & No. of seq.s & Sensitivity & Specificity & MCC & Average MCC & Overall Accuracy \\
\hline \multirow[t]{11}{*}{ Our method (239I sequences) } & chlo & 750 & 0.9733 & 0.8795 & 0.8893 & 0.8343 & 0.8703 \\
\hline & cyto & 432 & 0.9329 & 0.8258 & 0.8491 & & \\
\hline & cysk & 41 & $0.756 I$ & 1.0000 & 0.8677 & & \\
\hline & E.R. & 69 & 0.7391 & 0.9623 & 0.8395 & & \\
\hline & extr & 114 & 0.8246 & 0.7581 & 0.7797 & & \\
\hline & golg & 29 & 0.6207 & 0.9474 & 0.7645 & & \\
\hline & mito & 210 & 0.7857 & 0.9066 & 0.8303 & & \\
\hline & nucl & 456 & 0.8509 & 0.8788 & 0.8329 & & \\
\hline & pero & 52 & 0.7500 & 0.9512 & 0.8417 & & \\
\hline & plas & 165 & 0.7212 & 0.9675 & 0.8255 & & \\
\hline & vacu & 73 & $0.767 \mid$ & 0.9655 & 0.8569 & & \\
\hline $\begin{array}{c}\text { Horton et al. (2006) (2426 } \\
\text { sequences) }\end{array}$ & & & & & & & 0.86 \\
\hline
\end{tabular}

Note: In our method, although gap penalty $=0.4, \gamma=2.5, c=10, w=20$ are given, posconstraint and negconstraint are not specified. 
Table 4: Comparison of predictive accuracy for non-plant proteins in the TargetP data set. "mTP", "SP", and "other" indicate proteins destined for mitochondria, secretory pathway, and other locations (nucleus and cytosol), respectively.

\begin{tabular}{|c|c|c|c|c|c|c|}
\hline Predictor & Location & Sensitivity & Specificity & MCC & Average MCC & Overall Accuracy \\
\hline \multirow[t]{3}{*}{ Our method } & mTP & 0.7647 & 0.8990 & 0.8005 & 0.8452 & 0.9204 \\
\hline & $\mathrm{SP}$ & 0.9157 & 0.9056 & 0.8790 & & \\
\hline & other & 0.9576 & 0.9324 & 0.8560 & & \\
\hline \multirow[t]{3}{*}{ Matsuda et al. (2005) } & mTP & 0.8303 & 0.8635 & 0.8228 & 0.8542 & 0.9229 \\
\hline & SP & 0.9091 & 0.9118 & 0.8788 & & \\
\hline & other & 0.9498 & 0.9409 & 0.8609 & & \\
\hline \multirow[t]{3}{*}{ Kim et al. (2004) } & mTP & 0.6483 & 0.8569 & 0.7121 & 0.7635 & 0.8762 \\
\hline & SP & 0.8530 & 0.8736 & 0.8158 & & \\
\hline & other & 0.9389 & 0.8819 & 0.7626 & & \\
\hline \multirow[t]{3}{*}{ Emanuelsson et al. (2000) } & mTP & 0.89 & 0.67 & 0.73 & 0.8233 & 0.900 \\
\hline & SP & 0.96 & 0.92 & 0.92 & & \\
\hline & other & 0.88 & 0.97 & 0.82 & & \\
\hline \multirow[t]{3}{*}{ Reczko et al. (2004) } & $\mathrm{mTP}$ & 0.78 & 0.82 & 0.77 & 0.8333 & 0.913 \\
\hline & SP & 0.93 & 0.91 & 0.89 & & \\
\hline & other & 0.93 & 0.94 & 0.84 & & \\
\hline
\end{tabular}

Note: In our method, although gap penalty $=0.4, \gamma=2.5, c=10, w=20$ are given, posconstraint and negconstraint are not specified.

NNC. Details are shown in the first and third methods of Table 6.

Furthermore, according to Matsuda et al. (2005), the overall accuracy of NNC with Smith-Waterman score is 75.7 $\%$. Therefore, in the control experiment where the different similarity measures (our proposed method and Smith-Waterman score) and the same classifier (NNC) are used, our proposed similarity measure was better than Smith-Waterman score by 0.0558 .
Mitopred [31] is a web server which enables prediction of nucleus encoded mitochondrial proteins in all eukaryotic species. We input plant proteins of TargetP into Mitopred and compared the accuracies with our method. Table 7 shows the comparison of our method and Mitopred of each confidence cutoff. When confidence cutoff is $60 \%$, Mitopred yielded its best MCC of 0.7816 . However, our MCC is 0.8587 and higher than that of Mitopred by 0.0771 .

Furthermore, MitPred is a web-server specifically trained to predict the proteins which are destined to localized in

Table 5: Comparison of predictive accuracy for plant proteins in the TargetP data set with three types of feature vectors

\begin{tabular}{|c|c|c|c|c|c|c|}
\hline Predictor & Location & Sensitivity & Specificity & MCC & Average MCC & Overall Accuracy \\
\hline \multirow[t]{4}{*}{20 feature vectors (amino acid composition) } & cTP & 0.8440 & 0.9015 & 0.8507 & 0.8655 & 0.9096 \\
\hline & mTP & 0.9348 & 0.9125 & 0.8735 & & \\
\hline & SP & 0.9665 & 0.9319 & 0.9282 & & \\
\hline & other & 0.8148 & 0.8684 & 0.8095 & & \\
\hline \multirow[t]{4}{*}{400 feature vectors (all adjacent amino acid composition) } & cTP & 0.8227 & 0.4128 & 0.4806 & 0.6126 & 0.7372 \\
\hline & mTP & 0.8342 & 0.8797 & 0.7686 & & \\
\hline & SP & 0.8253 & 0.8880 & 0.8015 & & \\
\hline & other & 0.2963 & 0.8000 & 0.4339 & & \\
\hline \multirow[t]{4}{*}{40 feature vectors } & cTP & 0.8014 & 0.9040 & 0.8270 & 0.8594 & 0.9064 \\
\hline & mTP & 0.9402 & 0.9081 & 0.8739 & & \\
\hline & SP & 0.9628 & 0.9317 & 0.9255 & & \\
\hline & other & 0.8272 & 0.8590 & 0.8110 & & \\
\hline
\end{tabular}

Note: Parameters of Table 2 are used for all methods. 
Table 6: Comparison of predictive accuracy of our methods for plant proteins in the TargetP data set. "cTP", "mTP", "SP", and "other" indicate proteins destined for chloroplast, mitochondria, secretory pathway, and other locations (nucleus and cytosol), respectively.

\begin{tabular}{lccccc}
\hline Predictor & Location & Sensitivity & Specificity & MCC & Average MCC Overall Accuracy \\
\hline Our method (SVM with specifying posconstraint and & cTP & 0.8440 & 0.9015 & 0.8507 & 0.8655 \\
negconstraint) & mTP & 0.9348 & 0.9125 & 0.8735 \\
& SP & 0.9665 & 0.9319 & 0.9282 \\
& other & 0.8148 & 0.8684 & 0.8095 & 0.9096 \\
& cTP & 0.7518 & 0.9550 & 0.8249 & 0.8525 \\
\hline Our method (SVM without specifying posconstraint and & mTP & 0.9592 & 0.8506 & 0.8363 & \\
negconstraint) & SP & 0.9554 & 0.9554 & 0.9375 & \\
& other & 0.7963 & 0.8897 & 0.8111 & \\
& cTP & 0.7447 & 0.7664 & 0.7131 & 0.7255 \\
\hline Nearest Neighbor Classifier (with our similarity measure) & mTP & 0.8750 & 0.7854 & 0.7098 \\
& SP & 0.8848 & 0.9015 & 0.8508 & \\
& other & 0.6111 & 0.7674 & 0.6284 &
\end{tabular}

Note: In the first method, all parameters of Table 2 are given. In the second and third methods, gap penalty $=0.4, \gamma=2.5, c=10, w=20$ are given although posconstraint and negconstraint are not specified.

mitochondria in yeast and animals [32]. We applied MitPred to both plant and nonplant proteins of TargetP. MitPred has three types of methods; SVM method, BLAST+SVM method and HMM+SVM method. For both plant and nonplant datasets, BLAST+SVM method yielded the better MCC than any other method of MitPred. For plant dataset, MCC of BLAST+SVM method of MitPred was 0.8158 , which is less than MCC of our method by 0.0429. On the other hand, for nonplant dataset, MCC of BLAST+SVM method of MitPred was 0.9268, which is better than MCC of our method by 0.1642 . Since MitPred is specifically trained to predict the mitochondrial proteins in yeast and animals, the accuracy for nonplant mTP is better than ours. However, our accuracy is better than that of MitPred for plant although our prediction method is not specialized to mTP. Table 8 shows the detailed comparison of our method and MitPred.

By utilizing BLASTclust, we obtained non-redundant dataset at $70 \%$ and $25 \%$ from TargetP plant data set. Table 9

Table 7: Comparison of predictive accuracy of our method and Mitopred for plant proteins in the TargetP data set

\begin{tabular}{lcccc}
\hline Predictor & Location & Sensitivity & Specificity & MCC \\
\hline Our method & mTP & 0.9348 & 0.8958 & 0.8587 \\
Mitopred (60\%) & mTP & 0.9348 & 0.8132 & 0.7816 \\
Mitopred (85\%) & mTP & 0.5815 & 0.8807 & 0.5918 \\
Mitopred (99\%) & mTP & 0.0598 & 0.8148 & 0.1492 \\
\hline
\end{tabular}

Note: Parameters of Table 2 are used for our method. shows numbers of sequences for each location. By applying our SVM based method to these data sets, overall accuracy of 0.8625 and 0.7829 were obtained respectively. Therefore, it can be said that our method is efficient even when sequence similarity is relatively small. Details are shown in Table 10.

\section{Discussion}

We proposed a novel subcellular location predicting method which is based on sequence alignment and amino acid composition. Through fivefold cross validation tests for TargetP plant data sets, we obtained the overall accuracy of 0.9096 and the average MCC of 0.8655 . These values are higher than existing predictors which use only sequence information. We believe that the high accuracy attained by our method indicates that our alignment algorithm is automatically detecting signal sequences. Localization signals, such as the mitochondrial transit signal are highly diverse at the amino acid level, but share some features such as regions of positive charge or amphiphilic nature. Thus by aligning the amino acid compositions of small blocks, instead of individual amino acids, our technique may capture some features of localization signals such as transit signals.

As mentioned in the previous section, our method is effective for "cTP", "mTP" and "SP," but less effective for "other". The reason is discussed in the following. In general, proteins destined for chloroplast, mitochondria, and secretory pathway have signal sequences in their $N$ termini. On the other hand, proteins destined for nucleus 
Table 8: Comparison of predictive accuracy of our method and MitPred for both plant and nonplant proteins in the TargetP data set

\begin{tabular}{|c|c|c|c|c|}
\hline Predictor & Location & Sensitivity & Specificity & MCC \\
\hline Our method & plant mTP & 0.9348 & 0.8958 & 0.8587 \\
\hline MitPred (SVM) & plant mTP & 0.8234 & 0.8584 & 0.7418 \\
\hline MitPred (BLAST+SVM) & plant mTP & 0.9429 & 0.8422 & 0.8158 \\
\hline MitPred (HMM+SVM) & plant mTP & 0.8668 & 0.8484 & 0.7644 \\
\hline Our method & nonplant $\mathrm{mTP}$ & 0.7547 & 0.8333 & 0.7626 \\
\hline MitPred (SVM) & nonplant mTP & 0.8194 & 0.8863 & 0.8302 \\
\hline MitPred (BLAST+SVM) & nonplant mTP & 0.9380 & 0.9355 & 0.9268 \\
\hline MitPred (HMM+SVM) & nonplant mTP & $0.857 \mid$ & 0.9408 & 0.8830 \\
\hline
\end{tabular}

Note: In our methods, parameters shown in Table 2 of the manuscript were used, but "posconstraint" and "negconstraint" were not specified for nonplant mTP. For MitPred, we used default parameters as follows. In SVM method, threshold was set as 0.5. E-value cutoff of BLAST+SVM method was I e-4. E-value and SVM threshold for HMM based Pfam search+SVM method were I e-5 and 0.5 respectively.

and cytosol have one or more signal sequences in the middle part of their sequence. As explained later, global alignment is applied to left ends of sequences and local alignment is applied to right ends of sequences in our method as shown in Fig. 3. Then, it detects signal sequences in $N$ termini with higher probability than in the middle part of sequences. This is the reason why our method cannot improve the accuracy for "other" substantially. Furthermore, conventional local alignment, in which local alignment is applied to both ends of sequences, did not improve the prediction accuracy in our preliminary experiment. This fact is reasonable since conventional local alignment ignores signal sequences in $\mathrm{N}$ termini with higher probability than our method.

Although the above discussion can also be applied to SP and the "other" subset of non-plant datasets of TargetP, the result for non-plant mTP seems to be contradicting to this. We think the reason is either due to the influence of not using distance frequency [22], or that the composition of non-plant mTP sequences is only $13.6 \%$. Alternatively, there may exist some unknown signal sequences relating to subcellular location in $\mathrm{N}$-termini of non-plant mTP proteins. However, it should be noted that the difference

Table 9: Numbers of sequences of non-redundant datasets at $\mathbf{2 5 \%}$ and $\mathbf{7 0} \%$ obtained from TargetP plant data sets by utilizing BLASTclust

\begin{tabular}{lcc}
\hline Subcellular location & $\begin{array}{c}\text { No. of sequences (plant) } \\
\text { (non-redundant at 25\%) }\end{array}$ & $\begin{array}{c}\text { No. of sequences (plant) } \\
\text { (non-redundant at 70\%) }\end{array}$ \\
\hline Chloroplast(cTP) & 95 & 116 \\
Mitochondrial(mTP) & 200 & 314 \\
Secretory(SP) & 119 & 232 \\
Nuclear+cytosolic(other) & 125 & 138 \\
\hline Total & 539 & 800 \\
\hline
\end{tabular}

of accuracies between our method and [22] is very small $(0.25 \%)$.

Parameters in Table 2 were determined by trial and error approach, because it is very important to assign appropriate values to them. Since our feature vector consists of only amino acid composition, the information of subcellular location signals may be disappeared if $w$ is too large or too small. Even when appropriate $w$ is given, the information of signals may be divided and ignored if the edge of the window is in the middle of a signal sequence. However, such ignored signals can be found if $c$ is set appropriately. $c=w / 2$ is considered to be one of the best relationships between $c$ and $w$.

Assigning appropriate values to $\gamma$ and gap penalty is also important. As explained later, the pairing score in alignment of our method takes a positive value when two blocks are similar to each other. On the other hand, the pairing score takes a negative value when two blocks are not similar. However, if $\gamma$ is too large, the pairing score takes a negative value in most cases. On the other hand, if $\gamma$ is too small, the pairing score takes a positive value in most cases. Therefore, it is very important to set appropriate $\gamma$ since it determines the threshold of "whether blocks are similar to each other". The value of gap penalty also strongly influences the result of alignment. The detailed method of determining these parameters are described in the following.

Let $p$ represent the gap penalty. We examined $p=0,0.2$, $0.4,0.6,0.81 .0, \gamma=0.3,0.6,1.0,1.5,2.0,2.5,3.0,3.5$ and $(w, c)=(40,20),(30,15),(20,10)$ respectively. Among values above, $(p, \gamma, w, c)=(0.4,2.5,20,10)$ yielded the best accuracy. Since we did not examine so many values, the accuracy may be improved by further optimization for these values. We believe that further optimization for $(w$, $c$ ) is more hopeful than those for $p$ and $\gamma$ to improve the 
Table 10: Predictive accuracies of our method for datasets of Table 9

\begin{tabular}{lcccccc}
\hline Predictor & Location & Sensitivity & Specificity & MCC & Average MCC & Overall Accuracy \\
\hline Our method (25\%) & cTP & 0.7684 & 0.6577 & 0.6434 & 0.7068 & 0.7829 \\
& mTP & 0.7900 & 0.8404 & 0.7111 & & 0.7751 \\
& SP & 0.7311 & 0.9158 & 0.6976 & & \\
& other & 0.8320 & 0.7172 & 0.7937 & 0.8625 \\
Our method (70\%) & CTP & 0.6638 & 0.8750 & 0.7289 & 0.8006 & \\
& mTP & 0.9618 & 0.8118 & 0.9020 & \\
& SP & 0.9095 & 0.9505 & \\
& other & 0.7246 & 0.8475 & 0.7431 & & \\
\hline
\end{tabular}

Note: Parameters of Table 2 are used for all methods.

accuracy. It is to be noted that the same values were used for $(p, \gamma, w, c)$ in all the experiments in this paper.

In terms of "posconstraint" and "negconstraint", the first and second methods of Table 6 show the comparison of
- (1) our method with specifying "posconstraint" and "negconstraint,"

- (2) our method without specifying "posconstraint" and "negconstraint."

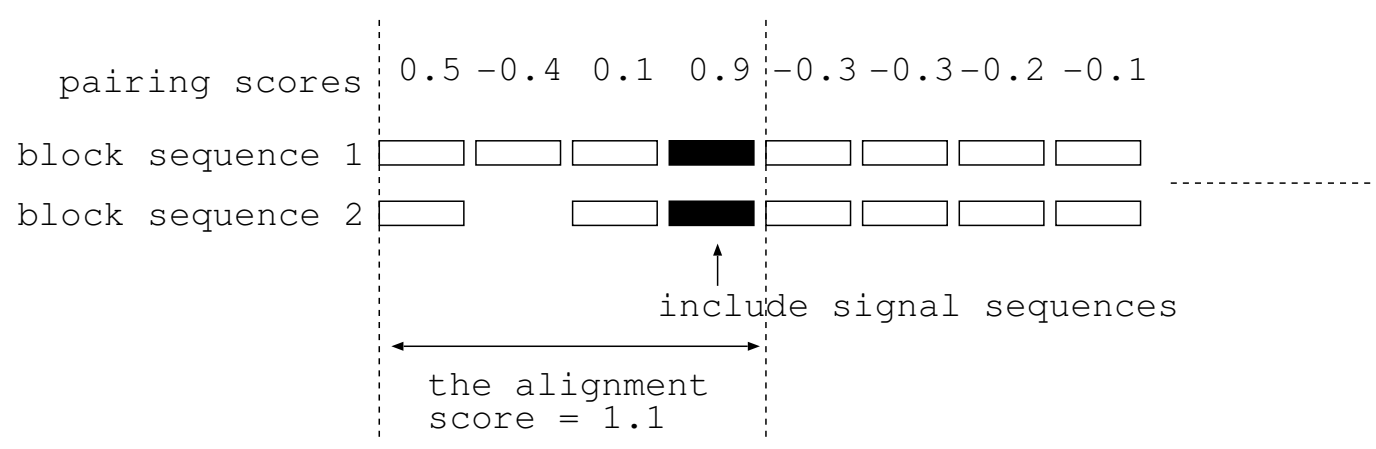

(A)

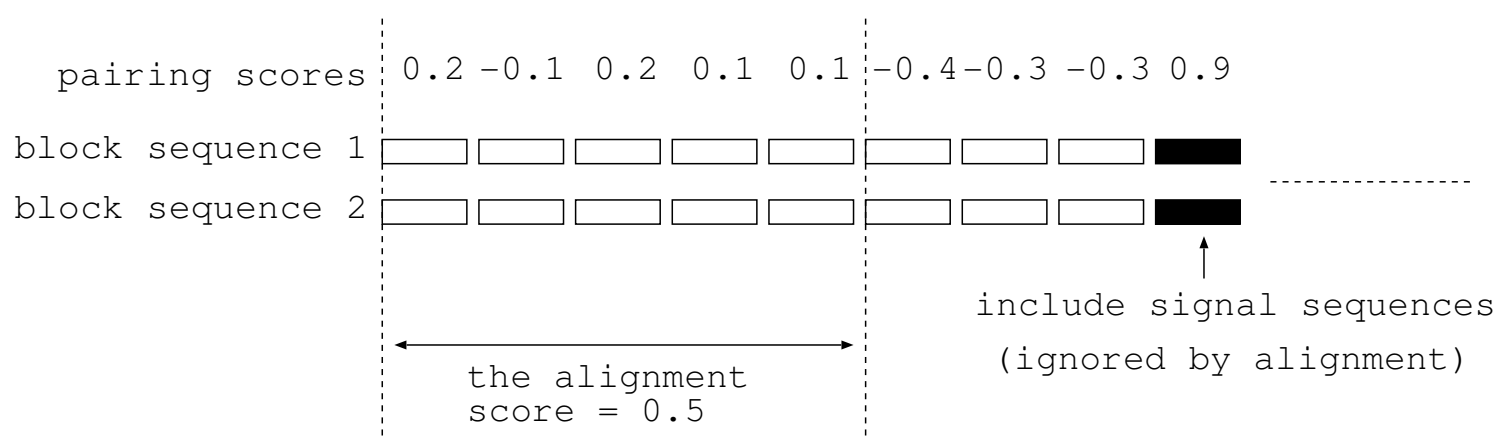

(B)

\section{Figure 3}

Global and local alignment. Global alignment is applied to left ends of block sequences and local alignment is applied to right ends of block sequences. (A) The alignment detects the signal sequence which is in the left part of sequences. (B) The alignment does not detect the signal sequence since the alignment score decreases if the block of the signal sequence is included. 
Although the average MCC and overall accuracy of (1) are better than those of (2) by 0.013 and 0.0107 respectively, (2) is better than the other predictors shown in Table 1. In terms of SP, MCC of (2) is better than (1) by 0.0093 although (1) is better than (2) for the other locations. Thus, our predictor can yield good accuracies even when "posconstraint" and "negconstraint" are not specified.

To optimize these parameters used in (1), we set posconstraint $=\exp (-0.07 \cdot i)$ and negconstraint $=\exp (-0.07 \cdot j)$ and scanned $i$ and $j$ from 0 to 99 . Then, values which yielded the best overall accuracy were used for (1) and are shown in Table 2. We believe that further optimization for "posconstraint" and "negconstraint" does not substantially improve the accuracies.

We also developed a web-based prediction system based on our proposed method for plant. It is available on [29]. When amino acid sequences in the FASTA format are given as shown in Fig. 4(A), the web-system returns the first and second candidates of the location and their scores as shown in Fig. 4(B). Although an overall accuracy of $90.96 \%$ was achieved in our five-fold cross validation tests, it takes about 10 seconds to predict a location. The reason why our web-system is not fast is that it calculates similarity scores between every input sequence and all training sequences. However, these calculations can be parallelized. If there are enough CPUs, the calculation time would be in a second although our web-system is not parallelized so far.

\section{Conclusion}

Our overall accuracy, average MCC and MCC for each location are higher than any other predictor which use sequence information for plant proteins of TargetP. Furthermore, our overall accuracy for plant proteins of WoLF PSORT is higher than WoLF PSORT although used datasets are slightly different. Therefore, it can be said that our prediction method for plant is efficient and succeeded in improving accuracies of existing methods. Although it is known that the overall accuracies (0.923) of the predictor for plant by Chou and Cai $[15,16]$ are slightly higher than those of our predictor, their method uses the information of gene ontology and functional domain. Since such information can be obtained only for known proteins, it is still important to develop a predictor which use only sequence information of amino acids. Thus, our predictor is considered to be useful for subcellular location prediction of newly-discovered proteins. Furthermore, the idea of combination of alignment and amino acid frequency is novel and general so that it may be applied to other problems in bioinformatics. Although our web-based predictor is now available for only plant, constructing the system for non-plant and plant which includes more numbers of subcellular locations is our on-going work.

\section{Methods}

Our proposed prediction method uses support vector machines (SVMs). In order to apply SVMs to classification of sequence data, it is usually required to define a kernel function between sequences. In our proposed method, the kernel value is the alignment score between two sequences. However, the alignment score is different from usual ones (e.g., Smith-Waterman score). We did not use the score matrix such as PAM or BLOSUM. In our proposed method, each input sequence is transformed into a sequence of blocks, where a block is a fixed-length consecutive substring of the input sequence. Then, in the alignment procedure, each block is treated as if it were a residue. The score between two blocks is calculated from features vectors of these blocks, where a feature vector of a block is based on the amino acid composition of the block.

Assume that sequence $1=A A A A A C C C C C D E F G H I I K K K L L$ $L L L$ and sequence $2=M M M M M C C C C C A A A A A C C C C C-$ NNN are given as shown in Figures 5(A) and 5(B) respectively. We transform sequence 1 (resp. sequence2) into a sequence of four blocks as in Fig. 5(A) (resp. Fig. 5(B)). Each block is of a fixed length $w$ and two consecutive blocks can overlap. The distance (in the number of residues) between two consecutive blocks is denoted by $c$. Note that $w=10$ and $c=5$ are used in Figures 5(A) and 5(B). In Fig. 5(B), " $\phi$ "s are assigned to the rightmost block since there are no corresponding amino acids. A feature vector is calculated for each block. Our feature vector consists of a composition of 20 amino acids. For example, the amino acid compositions for AAAAACCCCC, DEFGHI$I K K K$, and CCCCCNNN $\phi \phi$ are $(\mathrm{A}=5 / 10, \mathrm{C}=5 / 10$, the other $=0),(\mathrm{D}=1 / 10, \mathrm{E}=1 / 10, \mathrm{~F}=1 / 10, \mathrm{G}=1 / 10, \mathrm{H}=1$ / $10, \mathrm{I}=2 / 10, \mathrm{~K}=3 / 10$, the other $=0)$, and $(\mathrm{C}=5 / 10, \mathrm{~N}=$ $3 / 10$, the other $=0$ ) respectively in our method.

To calculate the alignment score, we have to define the score between blocks. For example, the score of alignment in Fig. 5(D) is calculated as the sum of the following.

- a pairing score between a gap and MMMMMCCCCC (a gap penalty)

- a pairing score between AAAAACCCCC and CCCCCAAAAA

- a pairing score between CCCCCDEFGH and AAAAACCCCC

We use $2 \cdot \exp \left(-\gamma\left\|\mathbf{b}_{x, j_{x}}-\mathbf{b}_{y, j_{y}}\right\|^{2}\right)-1$ as a pairing score between two blocks where $\mathbf{b}_{x, j_{x}}$ and $\mathbf{b}_{x, j_{x}}$ are feature vectors for blocks. Since $2 \cdot \exp \left(-\gamma\left\|\mathbf{b}_{x, j_{x}}-\mathbf{b}_{\gamma_{,} j_{y}}\right\|^{2}\right)-1$ always 


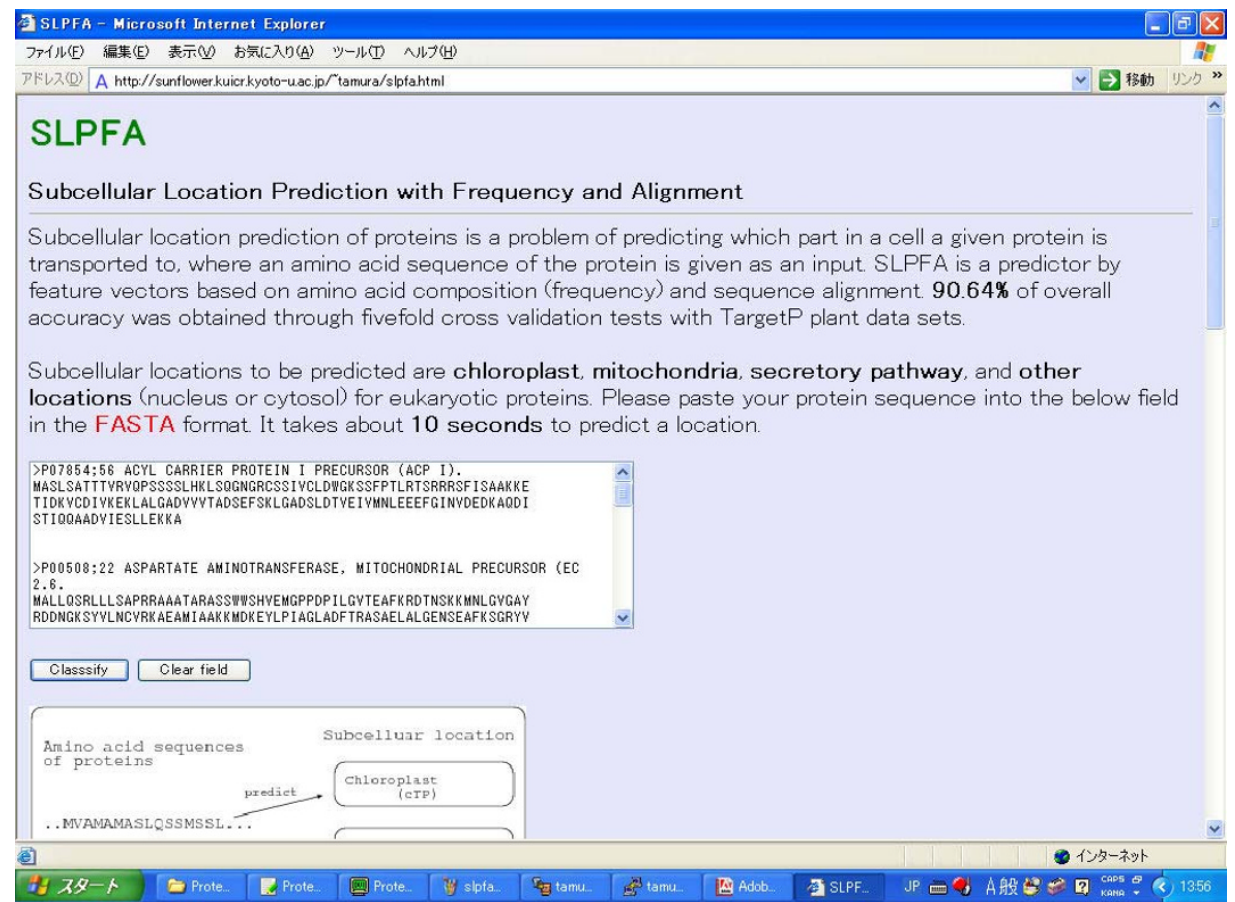

(A)

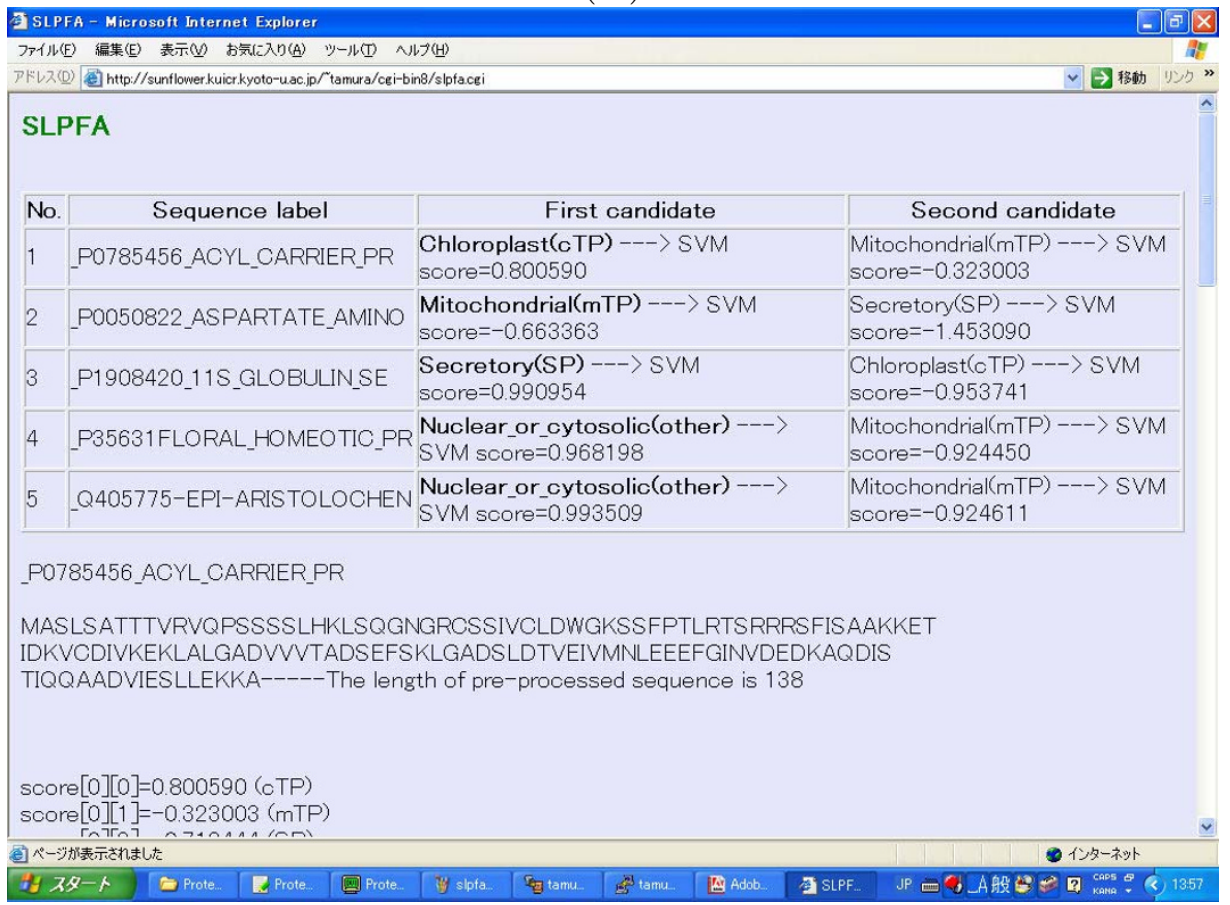

(B)

\section{Figure 4}

Web-based system. Our proposed method is implemented as a web-system and available on [29]. (A) Amino acid sequences in the FASTA format are pasted into our web-system as input. (B) Two candidate locations are shown for each input sequence along with scores. 


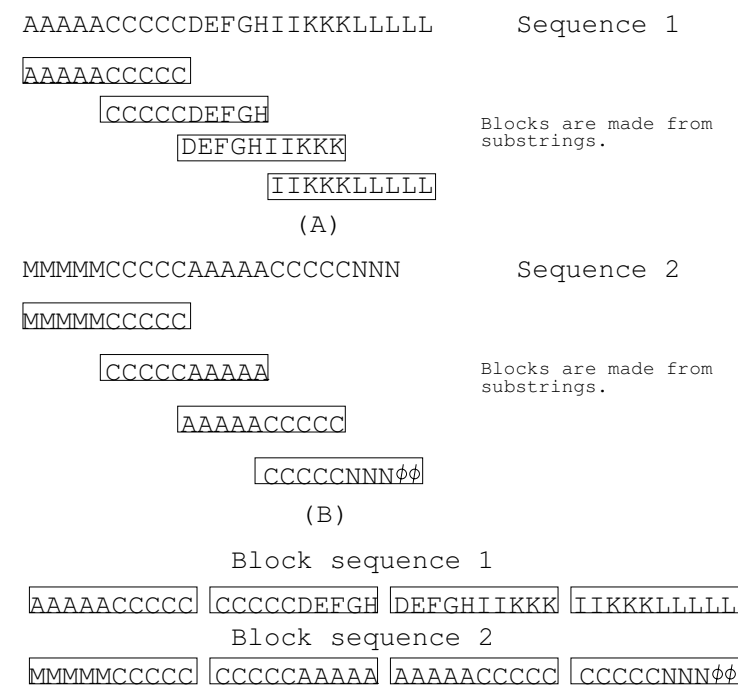

(C)

The alignment for sequence 1 and sequence 2

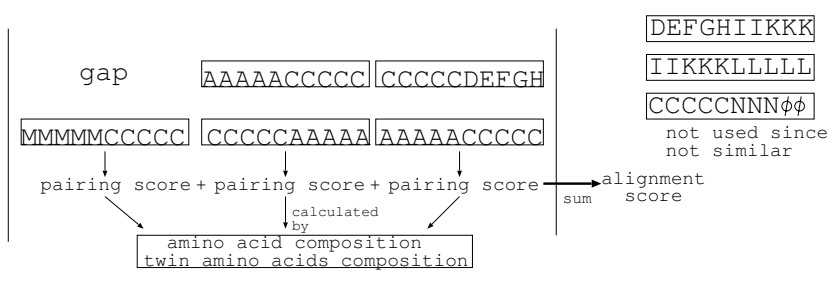

(D)

Figure 5

Alignment for block sequences. (A)(B)(C) Transformation of the input sequences into block sequences, where $w=$ 10 is the length of blocks and $c=5$ is the distance between neighboring blocks. (D) Alignment of block sequences. While global alignment is applied to left ends of block sequences, local alignment is applied to right ends of block sequences.

takes a value in $[-1,1]$, pairing scores also take a value in $[-1,1]$ in our implemented method. Note that the pairing score takes a positive value when two blocks are similar to each other. On the other hand, the pairing score takes a negative value when two blocks are not similar.

Finally, the highest alignment scores between every two block sequences are calculated by dynamic programming. In our method, while both left ends of block sequences must be used by the alignment, we do not have to use right ends of block sequences. In other words, global alignment is applied to left ends of block sequences and local alignment is applied to right ends of block sequences. For example, the best alignment between block sequences which are corresponding to sequence 1 and sequence 2 is shown in Fig. 5(D). Note that blocks
DEFGHIIKKK and IIKKKLLLLL of sequence 1 , and a block CCCCCNNN $\phi \phi$ of sequence 2 are not used in Fig. 5(D). Since $2 \cdot \exp \left(-\gamma|| \mathbf{b}_{x, j_{x}}-\mathbf{b}_{y_{1} j_{y}} \|^{2}\right)-1$ might take a negative value, there is a possibility that higher alignment scores are obtained in the case where right ends of sequences are not used than in the case where right ends of sequences must be used. For example, in Figures 5(C) and 5(D), pairing DEFGHIIKKK (or IIKKKLLLLL) and CCCCC$N N N \phi \phi$ takes a negative value in our implemented method since these are not similar to each other. The optimal scores of alignments are used as elements of the kernel matrix. Finally, our predictor selects a location whose "discriminant" value, which is calculated by "gist-classify" [33], is higher than any other location.

\section{Mathematical description of the method}

Here we present a mathematical description of our proposed method in order to provide precise information. Let $c$ and $w$ be some positive integers used as parameters. Let $S_{i}=s_{i, 1} s_{i, 2} \ldots s_{i, n_{i}} \phi \phi \phi \cdots$ be given protein sequences $(i=1$, $2, \cup, m)$, where $m$ is the number of sequences and $n_{i}$ is the length of $S_{i}$. Let $B_{i}=b_{i, 1} b_{i, 2} \ldots b_{i, \max }\left(\left[\left(n_{i}-w\right) / c\right\rceil, 0\right)+1$ be a sequence of substrings of $S_{i}$, where $b_{i, j}=s_{i, c(j-1)+1} s_{i, c(j-1)+2}$ $\ldots s_{i, c(j-1)+w}$. Note that there is a possibility that $b_{i, j}$ includes $\phi$.

Let $x$ and $y$ be integers which satisfy $1 \leq x, y \leq m$. Moreover, let $j_{x}$ and $j_{y}$ be integers which satisfy $1 \leq j_{x} \leq \max \left(\left[\left(n_{x}-w\right) /\right.\right.$ $c], 0)+1$ and $1 \leq j_{y} \leq \max \left(\left[\left(n_{y}-w\right) / c\right], 0\right)+1$. Feature vectors (defined later) of $b_{x, j_{x}}$ and $b_{y, j_{y}}$ are denoted by $\mathbf{b}_{x, j_{x}}$ and $\mathbf{b}_{y_{1} j_{y}}$ respectively. The kernel-like value between $B_{x}$ and $B_{\gamma^{\prime}}$ which is denoted by $K\left(B_{x^{\prime}} B_{y}\right)$, is calculated by the following dynamic programming (DP) procedure:

$$
\begin{gathered}
K\left(B_{x}, B_{y}\right)=\max _{j_{x}, j_{y}} D\left(j_{x}, j_{y}\right), \\
D\left(j_{x}, j_{y}\right)=\max \left\{\begin{array}{l}
\left.D\left(j_{x}-1\right), j_{y}\right)-p \\
D\left(j_{x}, j_{y}-1\right)-p \\
D\left(j_{x}-1, j_{y}-1\right)+f\left(j_{x}, j_{y}\right),
\end{array}\right.
\end{gathered}
$$

where $f\left(j_{x^{\prime}} j_{y}\right)=2 \cdot \exp \left(-\gamma\left\|\mathbf{b}_{x, j_{x}}-\mathbf{b}_{y, j_{y}}\right\|^{2}\right)-1, D(0,0)=0$, $D\left(j_{x^{\prime}}, 0\right)=-p j_{x^{\prime}} D\left(0, j_{y}\right)=-p j_{y^{\prime}} \gamma$ is the parameter of RBF kernel, and $p$ is the gap penalty of the alignment. Note that 
the value of $f\left(j_{x^{\prime}} j_{y}\right)$ lies in the interval $[-1,1]$. When $\mathbf{b}_{x, j_{x}}$ and $\mathbf{b}_{y, j_{y}}$ are similar, $f\left(j_{x^{\prime}} j_{y}\right)$ takes a positive value. Otherwise, $f\left(j_{x^{\prime}} j_{y}\right)$ takes a negative value.

The feature vector for representing a block is expressed by $\mathbf{b}=\left(r_{1}, r_{2}, \ldots, r_{20}\right)$, where $r_{1}, r_{2}, \ldots, r_{20}$ indicate the composition of 20 amino acids. Let score (cTP), score (mTP), score(SP), and score(other) be values of "discriminant" calculated for a protein sequence by gist-classify [33]. Our predictor selects $\max \{$ score $(c T P)$, score $(m T P)$, score $(S P)$, score(other) $\}$ and outputs the corresponding location. It is not guaranteed that the kernel matrix obtained from alignment scores is always valid (i.e., positive semi-definite). However, SVM training finished successfully in all cases of our computational experiments, which suggests that all the matrices used in the computational experiments can be treated as if it was positive semi-definite. Actually, in most cases, matrices produced by our method is not semi-definite since our method includes alignment. However, since absolute values of negative eigenvalues are small, SVM training can be executed successfully. If gap penalty and $\gamma$ in Table 2 are not appropriately given, we cannot train SVM since absolute values of negative eigenvalues are too large.

\section{Data sets}

The data sets used in this work were downloaded from TargetP [5] and WoLF PSORT [3]. TargetP data sets were collected from the SWISS-PROT database. Although TargetP data sets consist of plant and non-plant proteins, the mitochondrial proteins contain sequences from both plant and non-plant proteins since the number of plant mitochondrial proteins extracted from SWISS-PROT was too small to be used.

The redundancy reduced sets from which the training and test sets were built contain $141 \mathrm{CTP}, 368 \mathrm{mTP}, 269$ SP, and 162 other (nuclear or cytosolic) sequences for plant and $371 \mathrm{mTP}, 715 \mathrm{SP}$, and 1652 other (nuclear or cytosolic) sequences for non-plant as shown in Table 11.

Table II: Number of sequences in each subcellular location of TargetP plant and non-plant data sets

\begin{tabular}{lcc}
\hline Subcellular location & $\begin{array}{c}\text { No. of sequences } \\
\text { (plant) }\end{array}$ & $\begin{array}{c}\text { No. of sequences } \\
\text { (non-plant) }\end{array}$ \\
\hline Chloroplast(cTP) & 141 & - \\
Mitochondrial(mTP) & 368 & 371 \\
Secretory(SP) & 269 & 715 \\
Nuclear+cytosolic(other) & 162 & 1652 \\
\hline Total & 940 & 2738 \\
\hline
\end{tabular}

Matsuda et al. [22] checked the redundancy of this data set. They performed the prediction of subcellular location by using Smith-Waterman score, that is, the location of a sequence in the training data with a highest score is assigned to the corresponding query sequence in the test data. As a result, $75.7 \%$ and $84.0 \%$ of overall accuracies were obtained for plant and non-plant datasets respectively.

In order to perform a fivefold cross-validation test, each data set was partitioned into five subsets that have approximately equal sizes. Before partitioning, we randomly construct a permutation that consists of integers from 1 to 940 (or 2738) for plant (or non-plant). One subset is regarded as test data and the remaining four subsets as training data. This procedure is repeated five times so that each subset is used as test data once.

Plant protein data sets of WoLF PSORT are included in WOLF PSORT package version 0.2 that can be downloaded from [30]. By removing dual localization proteins, we obtained 2391 proteins in which 11 kinds of subcellular location exist. There was 35 proteins which have dual locations. Details are shown in Table 3.

\section{Accuracy measures}

In order to implement SVM, we used the software GIST [33]. We evaluated the prediction performance of our method by calculating sensitivity, specificity, Matthew's correlation coefficient (MCC) [34], and overall accuracy for each subcellular location. The definitions of these measures are as follows:

$$
\begin{gathered}
\text { Sensitivity }(l)=\frac{t p(l)}{t p(l)+f n(l)}, \quad \operatorname{Specificity}(l)=\frac{t p(l)}{\operatorname{tp}(l)+f p(l)}, \\
M C C(l)=\frac{\operatorname{tp}(l) \cdot \operatorname{tn}(l)-f p(l) \cdot f n(l)}{\sqrt{(t p(l)+f n(l))(t p(l)+f p(l))(\operatorname{tn}(l)+f p(l))(\operatorname{tn}(l)+f n(l))}}, \\
\text { Overall accuracy }=\frac{1}{m} \sum_{l=1}^{k} t p(l),
\end{gathered}
$$

where $m$ is the total number of protein sequences and $k$ is the number of subcellular locations. $t p(l)$ is the number of correctly predicted sequences belonging to location $l$ (true positive). $\operatorname{tn}(l)$ is the number of correctly predicted sequences that do not belong to location $l$ (true negative). $f p(l)$ is the number of overpredicted sequences in location $l$ (false positive). $f n(l)$ is the number of underpredicted sequences in location $l$ (false negative).

\section{Availability and requirements}

Project name: SLPFA; 
Project home page: http://sunflower.kuicr.kyoto-u.ac.jp/ ntamura/slpfa.html;

Operating system(s): Platform independent;

Programming language: none;

License: no restriction;

Any restrictions to use by non-academics: no restriction.

\section{Authors' contributions}

TT designed the detailed methods and algorithms, managed the computer experiment and constructed the webbased system. TA proposed the basic idea of the methods and supervised this work. Both authors read and approved the final manuscript.

\section{Acknowledgements}

We thank Dr. Morihiro Hayashida of Kyoto University for valuable comments and supports. This work was supported by "Education and Research Organization for Genome Information Science" with support from MEXT (Ministry of Education, Culture, Sports, Science and Technology).

\section{References}

I. Nakai K, Kanehisa M: A knowledge base for predicting protein localization sites in eukaryotic cells. Genomics 1992, |4:897-9||.

2. Horton P, Nakai K: Better prediction of protein cellular localization sites with the $\boldsymbol{k}$ nearest neighbors classifier. Proc Int Conf Intell Syst Mol Biol 1997, 5: I 47-I52.

3. Horton P, Park KJ, Obayashi T, Nakai K: Protein subcellular localization prediction with WoLF PSORT. Proc the 4th Annual Asia Pacific Bioinformatics Conference 2006:39-48.

4. Horton P, Park KJ, Obayashi T, Fujita N, Harada H, Adams-Collier CJ, Nakai K: WoLF PSORT: Protein Localization Predictor. Nucleic Acids Research 2007. doi:10.1093/nar/gkm259

5. Emanuelsson $O$, Nielsen H, Brunak S, von Heijne G: Predicting subcellular localization of proteins based on their $\mathbf{N}$-terminal amino acid sequence. J Mol Biol 2000, 300:1005-1016.

6. Nielsen H, Engelbrecht J, Brunak S, von Heijne G: Identification of prokaryotic and eukaryotic signal peptides and prediction of their cleavage sites. Protein Eng 1997, 10:1-6.

7. Emanuelsson O, Nielsen H, von Heijne G: ChloroP, a neural network-based method for predicting chloroplast transit peptides and their cleavage sites. Protein Sci 1999, 8(5):978-984.

8. Reczko M, Hatzigeorgiou A: Prediction of the subcellular localization of eukaryotic proteins using sequence signals and composition. Proteomics 2004, 4:159I-1596.

9. Cedano J, Aloy P, Perez-Pons JA, Querol E: Relation between amino acid composition and cellular location of proteins. J Mol Biol 1997, 266:594-600.

10. Chou KC, Elrod DW: Using discriminant function for prediction of subcellular location of prokaryotic proteins. Biochem Biophys Res Commun 1998, 252:63-68.

II. Reinhardt A, Hubbard T: Using neural networks for prediction of the subcellular location of proteins. Nucleic Acids Res 1998, 26:2230-2236

12. Hua S, Sun Z: Support vector machine approach for protein subcellular localization prediction. Bioinformatics 200I, 17:721-728.

13. Chou KC: Prediction of protein cellular attributes using pseudo-amino acid composition. Proteins 200I, 43:246-255.

14. Cai YD, Chou KC: Predicting subcellular localization of proteins in a hybridization space. Bioinformatics 2004, 20: I I5 I-I I 56.

15. Chou KC, Cai YD: A new hybrid approach to predict subcellular localization of proteins by incorporating gene ontology. Biochem Biophys Res Commun 2003, 3 I I:743-747.
16. Chou KC, Cai YD: Predicting subcellular localization of proteins by hybridizing functional domain composition and pseudo-amino acid composition. J Cell Biochem 2004, 91: I 197-I 203.

17. Park KJ, Kanehisa M: Prediction of protein subcellular locations by support vector machines using compositions of amino acids and amino acid pairs. Bioinformatics 2003, 19:1656-1663.

18. Yu CS, Lin CJ, Hwang JK: Predicting subcellular localization of proteins for Gram-negative bacteria by support vector machines based on $\mathbf{n}$-peptide compositions. Protein Science 2004, I 3:1402-I 406.

19. Yu CS, Wang JY, Yang JM, Lyu PC, Lin CJ, Hwang JK: Fine-grained protein fold assignment by support vector machines using generalized npeptide coding schemes and jury voting from multiple-parameter sets. Proteins 2003, 50:53I-536.

20. Guo J, Lin Y, Sun Z: A novel method for protein subcellular localization: Combining residue-couple model and SVM. Proceedings of the 3rd Asia-Pacific Bioinformatics Conference 2005:I I7-I 29.

21. Nair R, Rost B: Mimicking cellular sorting improves prediction of subcellular localization. Journal of Molecular Biology 2005, 348(I):85- 100.

22. Matsuda S, Vert JP, Saigo H, Ueda N, Toh H, Akutsu T: A novel representation of protein sequences for prediction of subcellular location using support vector machines. Protein Science 2005, I4:2804-28I3.

23. Leslie C, Eskin E, Noble WS: The spectrum kernel: a string kernel for SVM protein classification. Pac Symp Biocomput 2002:564-575.

24. Bhasin M, Reinherz EL, Reche PA: Recognition and classification of histones using support vector machines. Journal of Computational Biology 2006, I 3:102-1 I2

25. Ben-Hur A, Noble WS: Kernel methods for predicting proteinprotein interactions. Bioinformatics 2005, 2 I Suppl I():i38-i46.

26. Bhasin M, Garg A, Raghava GP: PSLpred: prediction of subcellular localization of bacterial proteins. Bioinformatics 2005, 2I( I 0):2522-2524.

27. Bhasin M, Raghava GP: ESLpred: SVM-based method for subcellular localization of eukaryotic proteins using dipeptide composition and PSI-BLAST. Nucleic Acids Research 2004, 32:W4|4-4I9.

28. Yu CS, Chen YC, Lu CH, Hwang JK: Prediction of protein subcellular localization. Proteins 2006, 64(3):643-65I.

29. Website SLPFA [http://sunflower.kuicr.kyoto-u.ac.jp/ tamura/ slpfa.html]

30. Website WoLF PSORT [http://wolfpsort.org/ aboutWoLF PSORT.html.en]

31. Guda C, Guda P, Fahy E, Subramaniam S: MITOPRED: a web server for the prediction of mitochondrial proteins. Nucleic Acids Research 2004, 32(2):W372-374.

32. Kumar M, Verma R, Raghava GPS: Prediction of mitochondrial proteins using support vector machine and hidden Markov model. Journal of Biological Chemistry 2005, 28 I:5357-5363.

33. Pavlidis P, Wapinski I, Noble WS: Support vector machine classification on the web. Bioinformatics 2004, 20(4):586-587.

34. Matthews BW: Comparison of the predicted and observed secondary structure of T4 phage lysozyme. Biochem Biophys Acto 1975, 405:442-45।.

Publish with Biomed Central and every scientist can read your work free of charge

"BioMed Central will be the most significant development for disseminating the results of biomedical research in our lifetime. "

Sir Paul Nurse, Cancer Research UK

Your research papers will be:

- available free of charge to the entire biomedical community

- peer reviewed and published immediately upon acceptance

- cited in PubMed and archived on PubMed Central

- yours - you keep the copyright

Submit your manuscript here:

http://www.biomedcentral.com/info/publishing_adv.asp

BioMedcentral 